\title{
EL DESARROLLO DE LA ACTIVIDAD TURÍSTICA EN CARTAGENA DE INDIAS: IMPLICACIONES SOCIALES Y URBANAS: 1943 - 1978
}

DEVELOPMENT OF TOURIST ACTIVTTY IN CARTAGENA: SOCIAL AND URBAN IMPLICATIONS. 1943- 1978

\author{
Por: Harold Carrillo Romero ${ }^{1}$ \\ Georgina Cabarcas Cañate \\ Adineth Vargas Hernández \\ Yusleinis Puello Pajaro
}

Artículo Recibido: 12 de septiembre 2012. Artículo Aprobado: 6 febrero 2013.

\section{RESUMEN}

Cartagena de Indias es la plaza turística más significativa del país. Sin embargo, es uno de los espacios con mayores índices de pobreza, exclusión y marginalidad social. El presente artículo es un acercamiento, desde una perspectiva socio-económica, a los procesos de instrumentalización de políticas sobre turismo en la ciudad. Enmarcado dentro del período de tiempo 1943-1978. Dicho esbozo, analiza el proceso y construcción histórica de Cartagena como ciudad turística, a partir de las legislaciones que se adelantaron para este fin y como en muchas medidas, este proceso catalizó signos de segregación y exclusión de los sectores populares de la ciudad, hacia zonas deprimidas y olvidadas.

Palabras Claves: Cartagena, Exclusión, Imagen Turística, Segregación, Turismo.

\section{ABSTRACT}

Cartagena de Indias is well known as the most significant tourist place in Colombia. However, it's one of the areas with the highest rates of poverty, social exclusion and marginalization. This article is an approach from a socio-economic perspective to the processes of manipulation of tourism polices in the city. This work is framed from 1943 to 1978, analyzing the process of a historic construction of Cartagena as a tourist city. This process catalyzed sing of segregation and as a result of the legislations that were conducted for its goal, popular sectors of the city suffered the exclusion to depressed and forgotten areas.

Keywords: Cartagena City, Exclusion, tourist image, Segregation, Tourism. 


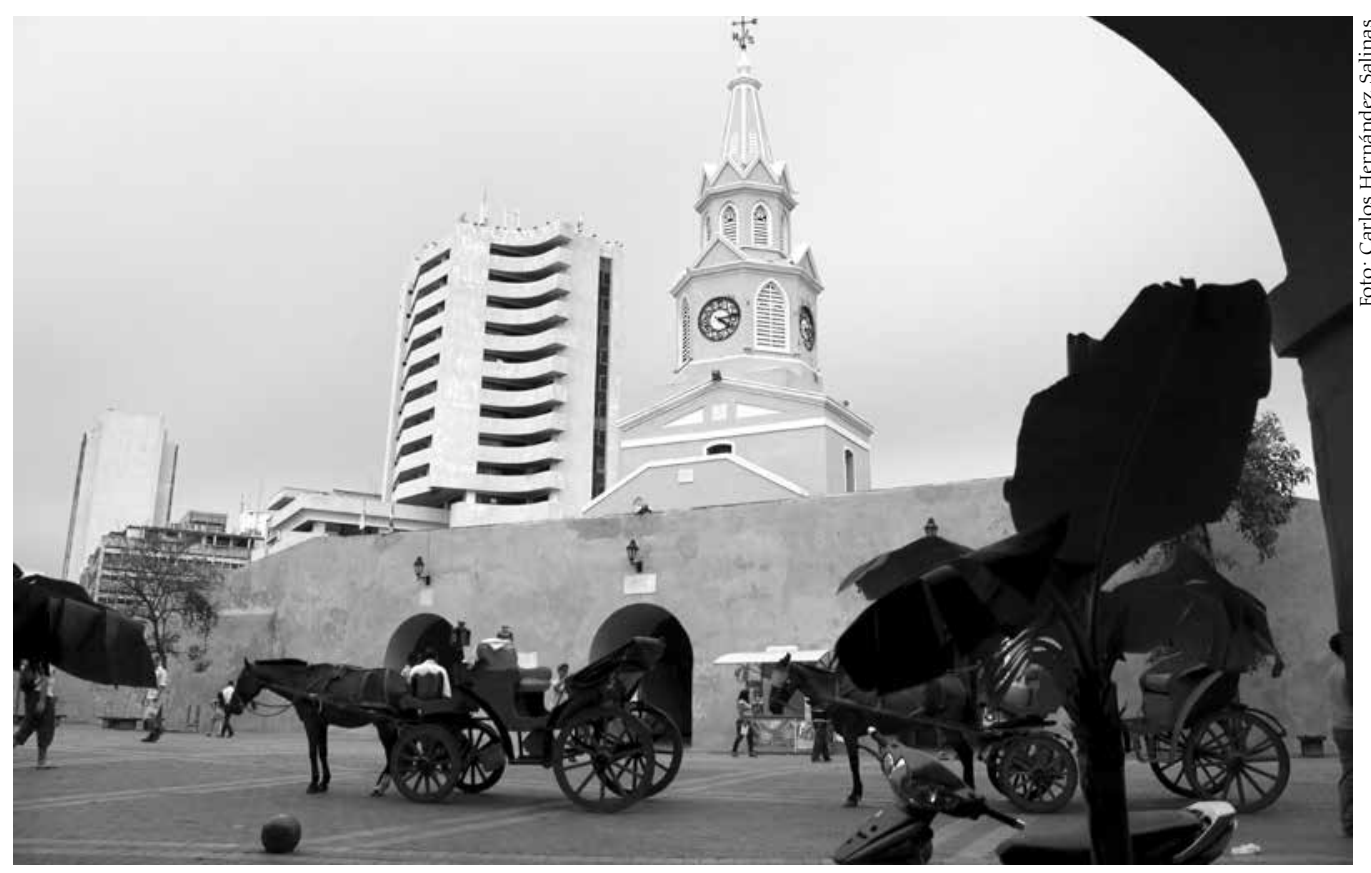

Plaza de los Coches 2013

\section{INTRODUCCIÓN}

Cartagena es una de las ciudades más importantes para el desarrollo del turismo colombiano. Desde los últimos 25 años la ciudad parece afianzar cada vez más su carácter turístico, el cual le ha permitido posicionarse en el país como uno de los destinos predilectos a la hora de pensar en vacaciones, recepciones y eventos de remembranza nacional e internacional. Festivales de todo tipo - música, arte, cine y literatura - al igual que premios, conciertos, concurso y hasta juegos, tienen lugar cada año en la ciudad. Esta cantidad de eventos donde casi siempre confluye el Jet Set criollo han elevado el nombre y la imagen de Cartagena como una de las ciudades más hermosas del continente, como así mismo una ciudad de puertas abiertas y donde la realidad social del país parece no haber permeado. Sin embargo, el 2 de junio de 2007 en el programa Fantasmas en la ciudad de Piedra de los populares reportajes del El mundo según Pirry, miles de colombianos se darían cuenta que Cartagena era espacio de contrastes sociales y urbanos. Guillermo Prieto (Pirry) le mostró a Colombia una Cartagena sin la murallas o sin las callejuelas y sus coloridos balcones; una ciudad lejos de lo que ilustran los folletos de promoción turística: por el contrario, se reveló el sinsabor del hambre, la pobreza, la violencia, la explotación sexual, las drogas y la exclusión. Desde esa polémica emisión, los ojos del país se concentraron en Cartagena, mientras que muchos de los cartageneros también empezaron a tomar conciencia de la disparidad entre la Cartagena que se muestra y la que se oculta. 
${ }^{2}$ Baumam, Zygmunt, La posmodernidad y sus descontentos. Madrid. AKAL Ediciones. 2001, p. 256.

${ }^{3}$ Méndez, Eloy, "Imaginario del lugar" en: Topofilia: Revista de Arquitectura, Urbanismo y Ciencias Sociales Número Especial Primer Colodel Turismo. México, 2008.

${ }^{4}$ Gisper, Carlos. "Las consecuencias sociales del turismo", en: Carlos Gisper (dir.), Enciclopedia Práctica Profesional de Turismo Hoteles y Restaurantes, Barcelona, Editorial. Océano, 1993 p. 153.

${ }^{5}$ Carrillo Romero, Harold El desarrollo de la actividad turística en Cartagena de Indias: Implicaciones Sociales y Urbanas. 1943-1978. Memorias VIII Encuentro Nacional de Estudiantes de Historia. Chicangana Yobenh (Compilador) Universidad Nacional Sede Medesidad Nacional Sede Medellín. ISBN 978-958-728 $042-5,2009$
Actualmente, esta situación no parece cambiar; por ejemplo, aún siguen siendo desplazados por los grandes hoteles muchos de los boquilleros, de igual forma, se han dado en concesión $12 \mathrm{~km}$ de playas e incluso, se ha estado pensando seriamente en trasladar de posición al actual mercado de la ciudad; medidas que naturalmente siguen con un deliberado proyecto de ciudad turística que busca mostrar a una Cartagena sin pobreza, miseria y sobre todo, sin sus gentes pobres. En este sentido, en el siguiente artículo se analiza el proceso y desarrollo de la actividad turística de Cartagena, para de esta manera entender, las implicaciones que ello ha generado en el contexto actual de la ciudad.

De acuerdo con el sociólogo Zygmun Bauman, quien considera que las consecuencias sociales del turismo son "procesos que se desprenden de las mismas implicaciones del capitalismo posmoderno ${ }^{2 \prime}$, podemos decir que la racionalización económica del turismo, conlleva a diseñar espacios exclusivos donde "se distribuyen la riqueza en base a un patrón que profundiza las desigualdades sociales mientras los turistas consumen el tiempo de ocio" ${ }^{3}$. De acuerdo a lo anterior, Carlos Gisper menciona al respecto lo siguiente:

En áreas menos desarrolladas, sin embargo, los enclaves turísticos pueden ser fuente de frustración y resentimiento al estar provistas estas pequeñas zonas frecuentadas por turistas con buenas carreteras, un sistema de abastecimiento de agua adecuado y demás servicios, mientras el resto de la comunidad permanece como siempre.

Un aeropuerto para jets en una isla del Caribe es magnífico, pero los nativos no pueden permitirse volar, como tampoco comer en los nuevos restaurantes o comprar en las boutiques. Los nativos que ganan unos ingresos marginales solo pueden observar, no pueden participar. Su situación cara a cara con el turista acentúa su pobreza y les puede conducir a la violencia ${ }^{4}$.

En ese sentido, afirmamos que procesos como los anteriormente citados, hacen que ciudades como Cartagena, se hayan convertido en un espacio diseñado para el consumidor más no para el ciudadano ${ }^{5}$.Medidas que abren paso a un carácter más fragmentado de la sociedad y del uso de los espacios públicos. Con base a lo anterior es pertinente describir cómo este proceso tuvo lugar en la ciudad de Cartagena. Para ello, hemos dividido el artículo en tres aspectos. El primero, estudia el proceso de inserción de Cartagena en las 
diferentes reglamentaciones nacionales a favor del turismo, y los antecedentes de la ciudad en el desarrollo de esta actividad. El segundo, examina el proceso de implementación de la industria turística en la ciudad y las políticas para ponerla en marcha, y por último, se analiza el impacto que tuvo en los sectores populares de la ciudad, la consolidación de una industria turística en Cartagena durante los años de 1943 a 1978.

\section{LOS ANTECEDENTES DE LA ACTIVIDAD TURÍSTICA EN CARTAGENA. AVATARES Y NORMATIVIDADES}

El panorama de las primeras décadas del siglo XX en Cartagena, nos pone de manifiesto en primer lugar, una ciudad en transición de recuperación económica y demográfica, inserta en los discursos de la modernidad y de la economía portuaria, que trata aceleradamente de recuperar el rótulo de puerto principal que le había expropiado Barranquilla.

Estos discursos, estuvieron acompañados de una serie de políticas de higiene, sanidad, expansión urbana, equipamiento e industria; donde sin duda, el eje principal sería el puerto y su conexión con el Caribe y el Canal del Dique (que a su vez se conectaría con el Río Magdalena), a través del ferrocarril Calamar Cartagena. En segundo lugar, una urbe estratégica e históricamente apta para el desarrollo del turismo, prueba de ello, fueron los vapores internacionales que desde 1909 empezaron a zarpar (aunque tímidamente) en territorio cartagenero. Al respecto, el periódico "El Porvenir" de Cartagena anotó: "según se nos informa tocará a nuestro puerto el vapor Avon de la Royal Mail, con cerca de 300 turistas", situación que también se informa el 9 de marzo de 1911 y en febrero de 1912, con el arribo del vapor Laurentic de la compañía White Starlie con 425 turistas $^{6}$.

A medida que se había experimentado una transitoria recuperación económica durante estas primeras décadas, también se hizo evidente un crecimiento poblacional en Cartagena ${ }^{7}$. Aquello obligaba a que el recinto urbano debía expandirse por fuera de las murallas, para que la ciudad, abriera las puertas a la modernidad. En parte este argumento justificó el derrumbamiento de fragmentos del cordón amurallado. No obstante, dicha medida generó controversias en unos sectores de la ciudad, pues la destrucción de las murallas, era un serio embate en contra del ornato y la herencia colonial de Cartagena. Sin embargo, para otros, las murallas representaban un impedimento para el desarrollo de la ciudad con miras a un progreso enmarcado dentro de la modernidad.

6 El Porvenir de Cartagena. Enero 26 de 1909. P. 3, marzo 9 de 1911. P. 1. febrero 4 de 1912, p. 1.

7 Adolfo Meisel afirma que la ciudad entre 1912 y 1951 obtuvo una tasa de poblamiento de $3,2 \%$, la más alta de toda su historia hasta ese momento. Véase Meisel Roca, Adolfo, "Cartagena, 1900 - 1950: A remolque de la economía nacional", en Calvo Stevenson, en Calu (comp.), Cartagena de Indias en el siglo XX, Bogotá, Banco
de la República, 2000, p. 23. 
8 Sierra, Germán, Marrugo Dennise y Quejada Pérez Raúl, La actividad turística en Cartagena de Indias, Editorial Universitaria, Universidad de Cartagena, Facultad de Ciencias Económicas 2004, p. 24.
En el marco de las más acaloradas discusiones acerca del futuro de las murallas, surgió la Sociedad de Mejoras Públicas (SMD) en 1923 como uno de los primeros intentos de un sector dirigente de Cartagena, por establecer políticas de preservación y fomento de las riquezas patrimoniales de esta ciudad. La constitución de este organismo, refleja entre otras cosas, el interés de comenzar a cimentar un emergente modelo de ciudad, orientado hacia la actividad turística. En este contexto, las autoridades municipales en compañía de la mencionada entidad, abrieron paso a diversas campañas, programas e inversiones, que posibilitaran la consolidación y puesta en marcha de un incipiente sector turístico. Para reafirmar lo anterior, el 2 de enero de 1924, el presidente del concejo de Cartagena, Antonio C. Ucrós, lanzó un proyecto para instaurar el premio al civismo en Cartagena, dicho galardón apuntó a que todos los ciudadanos tomasen una injerencia directa en las cuestiones que le pertenecían al bien público. Ese mismo año, a la referida entidad, se le otorga un soporte institucional importante por parte de la administración nacional, con la sanción de la Ley 32 de 1924 por la cual se le autorizó a la Sociedad de Mejoras Públicas, velar por la conservación de los monumentos históricos. Con la siguiente ley se finaliza la destrucción de las murallas.

Según lo anterior, podemos decir que la Sociedad de Mejoras Públicas, fue una de los organismos más importantes, de lo que podemos llamar, el primer momento del turismo cartagenero. Para la década de los 30, el Gobierno Nacional crea la ley 86 de 1931 donde se exhorta el fomento de la actividad turística. Esta legislación, contempla la creación de la Oficina Central de Turismo (OCT), la cual debía estar circunscrita al Ministerio de Industria y Comercio. Entre sus postulados podemos citar al respecto de la promoción de la actividad turística:

a.- Fomentar todo lo relativo al desarrollo turístico, como supervigilancia de las agencias de turismo, formación de itinerarios de excursiones, publicaciones de propaganda dentro y fuera del país, concursos para premiar las mejores obras de geografía, planos, mapas, historia, riquezas naturales, reseña de los lugares más apropiados para ser visitados, álbumes ilustrativos, etc., que sirvan para los turistas, y estudios para hacer conocer el país en forma clara, amena, sencilla y de fácil recordación ${ }^{8}$. 
La OCT al lado de la SMP, mancomunaron esfuerzos para el desarrollo del turismo y por medio de sistemas de difusión como la revista de la Cámara de Comercio y folletos de todo tipo, buscaron promocionar a la ciudad, resaltando los destinos y la belleza de la urbe . Aquello se había sustentado en el legado arquitectónico que tenían muchos de los inmuebles del centro de la ciudad, y de su cuidado y promoción, dependía el futuro de la Cartagena turística. A propósito, en septiembre de 1932 , bajo la Ley $11^{10}$, se crea en la ciudad la Junta Central de Monumentos Históricos y de Turismo, cuyo objeto era regular y aprovechar el valor patrimonial de Cartagena y su relación con el turismo. Todo ello se debía, al giro dinámico que adquirió esta empresa durante esta década, Meisel, citado por Sierra asegura que "entre 1935 y 1940 se recibieron 89.433 turistas en 641 vapores $^{11 "}$.

Para esta misma década, tienen lugar en la ciudad tres sucesos de gran importancia. El primero, es la apertura del moderno puerto de Manga, cuya obra remplazaría al antiguo muelle de la Machina localizado en Bocagrande; el nuevo muelle sumado a la navegabilidad del Canal del Dique, permitió acrecentar contundentemente el movimiento de carga y de pasajeros del puerto $^{12}$. Un segundo acontecimiento, es la inauguración del Reinado Nacional de Belleza, el cual benefició y promocionó la imagen de Cartagena como unos de los más representativos destinos turísticos del país; y por último, la erradicación de los tugurios de Pekìn, Pueblo Nuevo y Boquetillo. Al respecto, Adolfo Meisel afirma que "ya para 1937 había en estos tres barrios unas 400 casas, que el alcalde de la época, Daniel Lemaitre Tono, se puso en la tarea de erradicar y trasladar a sus vecinos al sector de Canapote en las faldas de la Popa" $^{13}$. En suma, se evidencia de este último acontecimiento, la intención de dar luz verde a la instauración de una imagen de destino turístico; la supresión de estos suburbios y sus traslados a la zona sur-oriental de la ciudad (zona despoblada y sin el acondicionamiento urbano necesario) va a significar el compromiso de crear y generar la sensación de una buena imagen turística.

El período de 1935 a 1942, concuerda con lo formado en este primer momento a la adecuación de infraestructura básica como transporte y hoteles, acciones que se van a comprobar con la reglamentación del servicio de autobuses de turismo, el oficio de equipajero, la inauguración del aeropuerto y la construcción del Hotel Caribe, con este último se marcó la expansión urbanoturística hacía el sector de Bocagrande y se abrió paso a que los dirigentes vean en otras zonas nuevos polos de desarrollo turístico como lo es el caso de la isla de Barú y la isla de Tierra Bomba.
${ }^{9}$ Claudia Vidal, Los Inicios de la Industria Turística en Cartagena de Indias, 1900 - 1950, Cartagena, Universidad de Cartagena, 1997, p. 117.

${ }^{10}$ A.H.C Diario Oficial. 23 de septiembre de 1932. Ley 11 de 1932, por la cual se crea en Cartagena la Junta de Monumentos Históricos y de Turismo.

${ }^{11}$ Sierra Anaya, Germán. Viajeros y visitantes - reseña histórica del turismo en Cartagena de Indias. Cartagena, Heliógrafo Moderno, 1998, p. 198.

${ }^{12}$ Germán Sierra Anaya, Dennise Marrugo Torrente y Raúl Quejada Pérez, Op. cit. 2004 . P. 26

${ }^{13}$ Meisel Roca, Adolfo, y Aguilera, María Modesta "¿la islas que se repite? Cartagena en el censo de Cartagena en el censo de población de 2005 , en: Documentos de trabajos sobre economía regional $N^{o}$ 109, Banco de la República, 2009 , p. 5. 


\section{LA CONSTRUCCIÓN DE LA CIUDAD TURÍSTICA: PROYECTOS Y CAMPAÑAS}

Al iniciarse la década de los 40, el turismo cartagenero nuevamente fue objeto de legislaciones que, en gran medida, reglamentaron el uso y desarrollo de esta actividad en el país. En este contexto, el Congreso de la República sanciona la Ley 48 de 1943, por medio de la cual, se fomenta el turismo en Colombia. Con dicha ley, se dio vía libre a la creación de la Dirección Nacional de Turismo como un ente encargado de diseñar políticas públicas dirigidas a desarrollar la producción turística en el territorio nacional, en especial en algunas ciudades con potencial para ello. Por tal motivo, se declara a Cartagena como primer centro turístico del país ${ }^{14}$, acontecimiento que marcó el hito para la puesta en marcha de una ciudad turística.

Esta legislación se dio como ampliación de la ley 86 de 1931. Dentro de las prerrogativas de la ley 48 se encontraba "a.- controlar todos y cada uno de los servicios que se brinden a los turistas o viajeros en hoteles, pensiones, y casas de hospedajes establecidas o que se establezcan en territorio de la República y vigilar por su estado sanitario, e imponer las sanciones a que haya lugar a los infractores"15.

Años siguientes, surgieron leyes encaminadas en dos direcciones. En primer lugar, las que se enfocan en la defensa del patrimonio colonial y su relación con el turismo, y en segundo lugar, aquellas que se encausan hacia la infraestructura para el desarrollo de esta empresa. Por consiguiente, la aparición de leyes como la 30 de $1943^{16}$, por el cual se restituye a la Sociedad de Mejoras Públicas la conservación y cuidado de un monumento histórico colonial - El Fuerte de San Felipe - , Ley 107 de 1946 ${ }^{17}$, Por la cual se reforman varios artículos de la $5^{\circ}$ de 1940 , sobre monumentos nacionales y la realización de algunas obras en la ciudad de Cartagena, Ley 71 de 1948 ${ }^{18}$, Por la cual se apropian unas partidas para la compra del Palacio de la Inquisición de Cartagena, y la Ley 163 de $1959^{19}$, por la cual se dictan medidas sobre

${ }^{14}$ Ibíd., p. 27.

${ }^{15}$ Ibíd., p. 27.

${ }^{16}$ A.H.C. Diario Oficial. 28 de octubre de 1943.

${ }^{17}$ A.H.C. Diario Oficial. 7 de enero de 1947

${ }^{18}$ A.H.C. Diario Oficial. 22 de diciembre de 1948.

${ }^{19}$ A.H.C. Diario Oficial. 30 de diciembre de 1959.

${ }^{20}$ A.H.C. Diario Oficial. 12 de diciembre de 1944.

${ }^{21}$ A.H.C. Diario Oficial. 20 de enero de 1946 defensa y conservación del patrimonio histórico, artístico y monumentos públicos de la nación, serían aquellas las encargadas de regular todo lo que le asistía al patrimonio colonial. En cuanto a infraestructura, leyes como la $8^{\circ}$ de $1944^{20}$, por la cual se ordena la celebración de una operación de administración y crédito, se conceden autorizaciones especiales al municipio de Cartagena y se dictan otras disposiciones, además la creación de la hoy Avenida Santander; la Ley 70 de $1946^{21}$, por medio de la cual se crea el 
Fondo Nacional de Turismo, y la Ley 60 de $1968^{22}$, por la cual se establecen estímulos a la industria turística. Las leyes aquí mencionadas, sirvieron de plataforma para los distintos proyectos que le sobrevenían a Cartagena en materia turística. Su viabilidad, sin temor a dudas, afianzó el sector turístico de la ciudad y generó un soporte legal capaz de garantizar el sostenimiento de dicho proyecto en el ámbito comercial y económico, pues, con la ausencia de este cuerpo de leyes, difícilmente, se hubieran establecido recursos públicos y privados, para la conformación de una estructura de servicios en aras de fortalecer la vocación turística - cluster - en la ciudad de Cartagena.

\section{Obras y proyectos para el desarrollo de la actividad turística en Cartagena}

Una de las más importantes obras de infraestructura que se levanta en la ciudad para estos años, es la construcción en 1941 del Hotel Caribe, fue el primero en la ciudad en ajustarse a características que requerían los estándares hoteleros internacionales, y además, con su inauguración en 1949, se comienza a marcar la expansión turístico - urbanística hacía el sector de Bocagrande $e^{23}$. La expansión hacia esta área, fue la base para que en los 60 los dirigentes de la ciudad, vieran en la zona norte de Cartagena, nuevos polos de desarrollo para el futuro de la actividad turística. En el año de 1955, la Junta de Turismo adelantó la ampliación de su Plan Turístico donde se cobijó la elaboración de una guía turística de la ciudad, control de tarifas, descripciones de los sitios de recreo y turismo, como también creación de programas de subsidio tanto para restaurantes, cafeterías, bares y grilles que posibiliten la prestación de una buena oferta turística. Entre los puntos que hacían parte del mencionado plan encontramos:

a) Dotación en Bocachica de refugios, cafeterías y facilidades para que el turista pueda pasar allí el tiempo que desee.

b) Subsidio a los restaurantes locales para que permanezcan abiertos hasta las 5:00 am, con lo cual se llenara toda una necesidad.

c) Dotación en las playas reservadas de Bocagrande de gimnasio, refrigerios, instructores para enseñanza de la natación y demás deportes acuáticos.

d) Construcción del embarcadero para el turismo que visita la bahía, en un lugar especial.

e) Establecimiento de servicio en el cerro de San Felipe para la venta permanente de comidas ligeras y otras comodidades para largas estadas. $^{24}$

${ }^{22}$ A.H.C. Diario Oficial. 26 de diciembre de 1968 .

${ }^{23}$ Samudio Trallero, Alberto, El crecimiento urbano de Cartagena en el Siglo XX: Manga y Bocagrande, Cartagena, Universidad Jorge Tadeo Lozano, 1999, p.50.

${ }^{24}$ A.B.N. El Universal. "Amplio plan de turismo está siendo elaborado por la Junta" Viarnes 25 de fer la de 1955, N 2077, p. 1 y 5. 
${ }^{25}$ Carrillo Romero, Harold, Ocultar la pobreza para inventar el paraíso: la construcción de la imagen turística de Cartagena 1953-1978. Tesis de grado para optar al título de historiador. Universidad de Cartagena. Marzo de 2011, p. 54.

${ }^{26}$ De León, Cebaldo y Pereiro, Xeraldo, La construcción imaginaria del lugar turístico: Kuna Yala. Universidad de Andalucía. España, 1995.
Para 1957, el alcalde de la ciudad Ernesto Carlos Martelo, creyó necesario que para el advenimiento de los nuevos desafíos del sector turístico, Cartagena debía, por lo menos, evaluar las condiciones del turismo que ofrece al mercado. Por tal motivo, se tomó la decisión de contratar a la firma Stanton Robbins \& Co. Inc., para que llevara a cabo un completo estudio acerca de las posibilidades turísticas de Cartagena. La evaluación de dicha firma se convirtió en el primer intento serio en consolidar la industria turística, siento quizás el trabajo más riguroso que se ha hecho de los problemas que posee este sector; en el cual se dejan ver dos necesidades: la primera, es la que tenía Cartagena de generar un turismo de calidad que estuviera de acuerdo a los estándares internacionales, para de esta manera poder entrar a competir con otros destinos del Caribe. La segunda, posesionarse como un sector productivo ante un país que para esta época, solo dependía de la exportación del café ya que constituían el 70\% de las exportaciones del país ${ }^{25}$.

De lo anterior, también queda en evidencia el deseo de los dirigentes por fortalecer una imagen turística, capaz de satisfacer las necesidades que el turismo internacional necesita para poder competir con otros destinos; la imagen puede lograr que el nombre de una ciudad o de un local turístico nos posicione positiva, negativa o indiferentemente frente a su realidad nominal en una realidad imaginada y representada ${ }^{26}$. En ese sentido, Cartagena direccionó todas sus políticas en la consecución de una representación espacial que caracterizará al turismo que se ofrece al visitante.

Este segundo momento, se caracterizó por varias coyunturas, por un lado, la nueva resignificación que adquiere el turismo como sector industrial en los mercados internacionales, lo que va a permitir que esta economía entre a dinamizar el panorama económico mundial; y por otro lado, ante tal coyuntura Cartagena se ve en la obligación de fomentar nuevas políticas para el desarrollo de esta industria, pero también se procura con ello, la obtención de una imagen (marca) turística que posibilite la promoción de los atractivos que ofrece la ciudad y la ayude a posicionarse en la economía internacional. Por tal motivo, el periodo de 1968 a 1982, va estar determinado por una serie de políticas públicas y proyectos tanto urbanos, sociales y culturales, para lograr un conjunto de representaciones espaciales que puedan promocionar el turismo cartagenero. En ese orden de ideas, el uso de los imaginarios urbanos jugaron un papel fundamental a la hora de generar y normativizar nuevos espacios para el desarrollo del turismo. Dichos imaginarios estuvieron enmarcados dentro de los discursos de la patrimonialidad de la urbe, por ello 
durante buena parte de los 60, encontraremos en la prensa de la época, una deliberada posición a favor de incitar en los cartageneros y extranjeros, el valor monumental y a la vez el perfil turístico de la ciudad.

Años más tarde, bajo la administración del Alcalde Lequerica tanto las autoridades locales y la Junta Promotora de Turismo, en aras de fortalecer el envío de recursos nacionales a la ciudad, propusieron la creación del Distrito Turístico de Cartagena. Tal como no los muestran las páginas del diario local El Universal de 3 de julio de 1963, según el alcalde en mención, con dicho proyecto se buscaba afianzar la ayuda y financiación por parte del Gobierno Nacional a los variados proyectos de cara al desarrollo de la actividad turística de Cartagena.

Es evidente que la proposición entraña un viejo anhelo de todos los colombianos que nos visitan periódicamente con el deseo de buscar esparcimiento y de renovar los sentimientos nacionales dentro de sus muros históricos. Por las razones dichas, (...) el proyecto no solo es de una alta conveniencia para los intereses locales, sino que corresponde a una aspiración colombianista que desea ver a Cartagena convertida en el mejor centro turístico del Caribe. ${ }^{27}$

Cabe destacar, que tanto los esfuerzos de la administración local y la entidad promotora de turismo estaban dirigidos a posicionar a Cartagena como centro turístico nacional de primer orden y por tanto, captar la atención del Gobierno Nacional era de vital importancia. La siguiente cita nos ilustra sobre ello:

Somos justos en considerar que las reservas de que dispone la empresa nacional de turismo, con todo y la diaria contribución de los hoteles, no son suficientes para corresponderle en ayuda a todos los festivales que hoy se realizan en el país. Para esto se necesitarían millones. Y por ello hemos venido sugiriendo desde hace días, que la empresa proceda a organizar un plan piloto de turismo, referido a una, dos o tres ciudades, aquellas que mejor se presten al fomento del ramo en gran escala. Concentrados los esfuerzos y la mayor parte de los recursos en la ejecución de ese plan, se evitaría la dispersión de auxilios, que minimizan los fondos disponibles sin que se alcancen los fines perseguidos para ninguna ciudad. Hasta hoy lo que se ha visto es la quemazón de esos auxilios mínimos en fiestas transitorias. (...) esta es la hora,

${ }^{27}$ ABN. El Universal" Para atraer el turismo internacional se propone crear distrito turístico" Miércoles 3 de junio de 1963, p. 1 y 8. 
por ejemplo, que Cartagena carece de un muellecito para embarque del turista a sus paseos de la bahía, carece de fondos con que embellecer sus monumentos históricos y sanear sus murallas (...)

Dentro de este contexto, encontramos que los planes turísticos están orientados hacia un incremento tanto del turismo local como internacional; por lo cual se alude a las principales atracciones de la ciudad, eventos, festividades y lugares como estrategia para impulsar el número de visitantes en esta plaza. Iniciativas como el acercamiento entre Barranquilla, Santa Marta y Cartagena como forma de viabilizar el turismo costeño nos ponen en escena la importancia que tenía para los empresarios de la ciudad lograr posesionar a Cartagena dentro de la dinámica turística nacional e internacional. En el mencionado acuerdo, los gobernantes y las entidades de fomento turístico de las respectivas ciudades declararon que "la Costa Atlántica es la más importante unidad turística de Colombia y que sus principales ciudades se complementan recíprocamente, por lo que se dará comienzo a un plan de actividades coordinadas estrechamente entre las oficinas de turismo de Santa Marta, Barranquilla y Cartagena"28. Por tal motivo, a partir de este momento se concentraron esfuerzos por parte de estas ciudades costeñas por posesionarse como centros turísticos de la región.

Fortalecer el turismo local, fue uno de los puntos que concentró la atención de la Junta Promotora de Turismo de Cartagena que a través de la publicidad y difusión de los eventos y festividades llevadas a cabo a lo largo del año, se buscó fomentar la constante llegada de visitantes. La creación de un Departamento de Promociones, fue una de las propuestas planteadas por el Gobierno Local, para que dicha entidad velara por el buen funcionamiento y organización de lo que al sector turístico y atención al turista se refiere:

Deberá funcionar "El Departamento de Promociones" para iniciar un plan de atracciones turísticas de las ciudades del país hacia Cartagena especialmente en los meses en que se desarrollan aquí certámenes de importancia nacional como Concurso de Belleza, festivales musicales, Festival de Cine. ${ }^{29}$

${ }^{28}$ ABN. Diario de la Costa "Reunión tripartita del turismo costeño" Martes 6 de
febrero de 1962 , p. 1

${ }^{29}$ ABN. EL Universal. "Vasto plan para incrementar el turismo local propone Martelo". Miércoles 17 de abril de 1963, N 5436, p. 1.
Ese mismo año, la Promotora de Turismo de Cartagena, solicitó a la Empresa Colombiana de Aeródromos (ECA), una autorización para llevar a cabo la construcción de una oficina de turismo en las instalaciones del aeropuerto de la ciudad, que facilite la información al turista sobre hoteles, atractivos 
turísticos, precios, etc. Todo esto orientado al mejoramiento y calidad del servicio brindado a los diferentes visitantes que arriban a esta plaza, que en muchas ocasiones, no contaban con el personal idóneo que les indicase y le suministre toda la información turística necesaria:

Esta oficina va a servir para orientar al turista respecto a los hoteles donde debe llegar, sitios que son de atracción, precios de artículos, carreras de automóviles, etcétera, etcétera. Pero es el caso que la ECA, dicen los informantes, no se han pronunciado sobre lo que se viene relatando creando una situación grave que va en contra de los intereses del turista pues llega desorientado a la ciudad sin poder contar con una persona que le indique y le suministre todo cuanto desea saber. ${ }^{30}$

Las diferentes iniciativas y proyectos destinados al mejoramiento de la actividad turística apuntaron principalmente hacia la promoción y fomento del turismo en esta plaza. Es así, que se hizo alusión constantemente a sitios, reformas e inversiones que posibiliten cautivar a gran afluencia de turistas, ejemplo de lo anterior, lo observamos en la siguiente cita:

Una vasta campaña para incrementar el turismo en Cartagena está adelantando la oficina de turismo de la ciudad en todos los frentes. Las campañas intensivas se están realizando a través del país y en ella se invita a todos los colombianos a que visiten y disfruten, dentro de sus históricas reliquias, de unas agradables vacaciones y de un ambiente sin igual. Una de las promociones se orienta a que los industriales inviertan en Cartagena, la ciudad del futuro. Y como una contribución al desarrollo turístico de Cartagena de Indias, se ha proyectado la Urbanización El Laguito, exclusivamente turística y situada en el elegante barrio de Boca Grande, al lado del Hotel Caribe. Esta nueva realidad de la ciudad está sobre una hermosa península acariciada por la brisa del mar y bañada por un espléndido sol. La Urbanización el Laguito esta llamada a convertirse en el Centro Turístico de Colombia. Su especial ubicación es, por si sola, una atracción para los inversionistas. El sitio, por su belleza natural, el mar que lo rodea, su privilegiada localización dentro de la ciudad, la facilidad de embarcadero propio de su yate, el fácil acceso a los mercados, comercios, restaurantes, grilles, casinos y a solo 50 minutos en jet 
desde Bogotá, son poderosas razones para que atraiga a los miles de turistas que desean pasar en la histórica ciudad unas alegres vacaciones. ${ }^{31}$

Otros mecanismos utilizados para fomentar la difusión de los atractivos turísticos de la ciudad fueron a través de la visita de periodistas y agentes extranjeros. Tal es el caso de la visita de dos agentes de viajes norteamericanos invitados por la Empresa Colombiana de Turismo, para que conocieran y a la vez, disfrutaran de las bellezas de nuestro país. Esta forma de extensión publicitaria le posibilitó a la ciudad dinamizar su potencial turístico.

Hay que destacar la importancia de la visita de los agentes de viaje norteamericanos que actualmente se encuentran aquí, por ser la primera vez que se reúnen un capítulo de ASTA en Colombia, $y$ en realidad, fueron dos, uno de la zona del oeste medio y el otro, de la parte norte de Nueva York. Ambos efectuaron sus deliberaciones en Bogotá y un capitulo vino a Cartagena donde las continuaron y el otro siguió hasta Quito, Ecuador. La importancia de esta visita como lo expresó anteriormente, se debe a que es la primera vez que se efectúa y que además estos señores son los que mueven el turismo de su país hacia las diversas partes del mundo. Ellos han quedado muy bien impresionados con Colombia y han manifestado que harán promociones para atraer turismo a nuestro país ${ }^{32}$

Por otro lado, uno de los eventos que viabilizó una mayor planeación y desarrollo de la actividad turística en Cartagena; fue la realización del Seminario turístico organizado por la ANDI en el año 1968, en el cual se trataron temas relacionados a la restauración de fortificaciones, construcción de vías, hoteles erradicación de tugurios, entre otros problemas y proyectos necesarios para el fomento y desarrollo del turismo en la ciudad. Entre los temas debatidos encontramos:

${ }^{31}$ ABN. "Campañas para e fomento turístico en Cartagena", El Universal. Marte 17 de enero de 1967, p. 9.

${ }^{32}$ ABN, "Plan de Turismo para Cartagena", El Universal. Viernes 3 de mayo de $1968, \mathrm{~N}^{\circ} 7000$, p. 1-11.
Exenciones y estímulos tributarios y cambiarios, pavimentación, alcantarillado, acueducto y energía, edificios del aeropuerto y pistas del mismo, Avenida Santander, defensa de las playas, dragado de los caños, ciénagas y avenidas circundantes, hotel de primera categoría, restauración de fortificaciones, mercado, parques y zonas aledañas para embarcaderos turísticos, erradicación de 
tugurios desde Chambacú hasta el aeropuerto, remodelación de casas coloniales, despeje del Fuerte de San Felipe a partir del puente de Heredia, remodelación de iglesias y conventos, construcción del circo de toros, buses de turismo y lanchas, zona de desarrollo futuro, adecuación de la ciénaga de la Virgen, ampliación en curso y proyectadas de teléfonos, delimitación de zonas de pesca y caza. ${ }^{33}$

Todas estas reflexiones que tiene lugar en dicho evento, abren paso a un amplio periodo de mejoramiento de las condiciones turísticas de la ciudad y los servicios que se ofrecen a los visitantes en esta plaza. Tal es el caso del proyecto de construcción de un centro Internacional de Turismo en la ciudad, pues era de utilidad, en palabras de Antonio Dáger Gerala, Gerente de la Promotora de Turismo, "dotar a Cartagena de un centro en donde puedan llevarse a cabo certámenes de gran magnitud como ocurre en Filadelfia, Nueva Orleans y otras grandes urbes" ${ }^{\prime 34}$. Con la creación y ejecución de estas campañas, los dirigentes pro-turísticos de la ciudad, aseguraban el mantenimiento de estos inmuebles, y además educarían a los cartageneros para que emprendieran la exclusividad que estos lugares tenían para el desarrollo turístico, aquello era entendido a la opinión pública como la "conciencia turística". Finalizando la década de los 60 , el turismo en la ciudad era ya uno de los sectores de mayor dinamismo, era frecuente ver en la prensa titulares que anunciaran la llegada de buques de Alemania, Italia, o los Estado Unidos. Sin embargo, los problemas fiscales y presupuestales siempre fueron constantes, al igual que los sectores populares de la ciudad, ya que ponían al descubierto los contrastes y ambigüedades del mito con el que se tejió el discurso turístico.

\section{IMPLICACIONES URBANAS Y SOCIALES DEL TURISMO EN CARTAGENA}

De la mano del crecimiento demográfico y urbano que sufrió la ciudad durante las primeras dos décadas del siglo XX, tuvo lugar la aparición de "invasiones" o tugurios justo al pie de la muralla; se trataban de improvisados barrios cuya estructura descansaba sobre los muros de los baluartes coloniales. Pekín, Pueblo Nuevo y Boquetillo, fue el albergue de pescadores artesanos, lo que constituía el refugio de sectores populares propensos al rechazo del incipiente sector turístico. En este sentido, la proliferación de barriadas tan cerca al centro de la ciudad, y peor aún ancladas en uno de los principales atractivo turísticos, van a significar una de primeras segregaciones urbanas que sufren los cartageneros en el siglo XX. Al respecto Carmen Cabrales menciona que "el proceso de poblamiento se inicia en esta zona en los años

${ }^{33}$ ABN. Diario de la Costa. "Un seminario sobre el turismo auspiciará ANDI en Cartagena", Jueves 10 de octubre de 1968, p. 3.

${ }^{34}$ A.B.N. El Universal. "Centro turístico tendrá Cartagena", Miércoles 24 de diciembre de 1968, N 8282, p. $1-12$. 
35 Cabrales, Carmen, Los barrios populares de Cartagena. En: Cartagena de Indias en el siglo $X X$. Calvo, Haroldo, Meisel, Adolfo (Edit.) Cartagena, Banco de la República. 2000, p. 188.

${ }^{36}$ Meisel Roca, Adolfo, y Aguilera, María Modesta, Op.Cit, p. 5.

${ }^{37}$ Orlando De Ávila, Políticas urbanas, pobreza y exclusión social en Cartagena: El caso de Chambacú 1956 - 1971 Cartagena, Universidad de Cartagena, Tesis para op-

tar a titulo de historiador.

tar a titulo de historiador.

Mayo de 2008, p. $60-90$ Tesis para optar a titulo de historiador. p. 36.
20 con la reubicación estratégica de los pobladores que habitaban Boquetillo, Boquerón, Pueblo Nuevo y Pekín, ubicados en los alrededores de las tenazas para el sitio que hoy es el barrio Canapote ${ }^{35 "}$. Lo anterior, podemos reafirmarlo reiterando lo ya citado por Adolfo Meisel, quien asegura que "ya para 1937 había en estos tres barrios unas 400 casas, que el alcalde de la época, Daniel Lemaitre Tono, se puso en la tarea de erradicar y trasladar a sus vecinos al sector de Canapote en las faldas de la Popa" ${ }^{36}$.

Esta primera erradicación de tugurios populares no es un hecho aislado a la forma en la que la ciudad se estaba proyectando; al igual que otros traslados, los habitantes fueron ubicados en zonas estratégicamente diseñadas para el plan de la ciudad turista, pues a los segregados, se les confinó en sectores distantes al centro turístico, en lugares sin el acondicionamiento urbano básico y además conservando el mismo precario ambiente de su antiguo entorno.

Otro de los barrios que germinó cerca al centro "patrimonial" de la ciudad, fue el barrio de Chambacú, del cual Orlando Deávila afirma que "para finales de los años 20, llegaron sus primeros pobladores provenientes de islas extremadamente cercanas a la ciudad o de barrios circunvecinos, como Bocachica y Torices, respectivamente ${ }^{37 \prime \prime}$. El problema de Chambacú, como lo llamó a menudo la opinión pública de Cartagena, fue y es uno de los símbolos más visibles de la segregación urbana en la ciudad, provocada en muchos aspectos, por el proyecto turístico de la urbe. Por un lado, la ciudad patrimonial se erigía con majestuosidad, y por otro, la miseria, la insalubridad y la pobreza de los cartageneros, echaban abajo el discurso turístico. Este paralelo entre el discurso turístico y los contrastes sociales de la cuidad, no era apto para la vista de los turistas. De fondo se encontraba el obstáculo que representaba a Chambacú para la imagen turística. Sin embargo, los imaginarios que se adoptaron para justificar en gran medida la erradicación de este barrio, fueron las antihigiénicas condiciones en la que se encontraba, la criminalización y estigmatización de sus habitantes, como gentes altamente peligrosas para el resto de los cartageneros. Al menos así, lo deja ver el Diario de la Costa el decir que:

Chambacú sigue siendo el clásico lunar de Cartagena. Mientras la ciudad tenga dentro de su perímetro esa "Zona negra" que es Chambacú estaremos muy lejos de haber entrado en una etapa en que la higiene y el aseo correspondan a las verdaderas necesidades que tiene nuestra urbe. De ahí que a esta hora muchos cartageneros 
vean con escepticismo una solución cercana a este magno problema que urge solucionar. Pues en verdad, los planes de redención de los habitantes de Chambacú marchan a paso de ganso, y quizás este año terminará sin que los ocupantes de esa Zona Negra lo hayan abandonado. ${ }^{38}$

Con noticias como estas también se desencadenaron imaginarios urbanos que satanizaban a todos los chambaculeros, es decir cada vez que se emitían noticias como redadas, allanamientos, desorden, hurtos y peleas, se trató de señalar negativamente a todos los habitantes del barrio, para de esa manera crear en los cartageneros la sensación de miedo y constante amenaza para la seguridad pública ${ }^{39}$.

Luego de muchos trámites con el Instituto de Crédito Territorial y las Empresas Públicas de Cartagena, se logró en 1971, trasladar hacia las goteras de la ciudad a los habitantes de la "Zona Negra de Chambacú" a barrios como República de Venezuela, Chiquinquirá, el Nuevo Porvenir, entre otros, donde seguirían guardando los mismos problemas con los que se justificó su erradicación.

En términos generales, la situación social y económica de los chambaculeros no había variado sustancialmente. La pobreza, que fue en principio lo que el gobierno decía querer erradicar, seguía su curso ininterrumpido. No podía esperarse un resultado distinto. Las causas originarias de la pobreza jamás fueron tocadas. La pobreza va más allá de la no posesión de vivienda, por lo tanto demanda soluciones estructurales que para el caso de Chambacú no fueron tomadas ${ }^{40}$.

Tras de la erradicación del barrio de Chambacú, aún quedaba otro problema igual de preocupante para el futuro de la ciudad, se trataba del Mercado Público de Getsemaní. Con la puesta en marcha del "Plan de acción para el proyecto de desarrollo turístico de la Costa Atlántica y San Andrés", realizado por la Corporación Nacional de Turismo de Colombia en 1972 se especificaba claramente que:

Otra componente de la infraestructura externa para el proyecto del turismo de Cartagena, es la remodelación urbana de algunas zonas de la ciudad que necesitan la eliminación de áreas deterioradas, de congestiones de tráfico y de tugurios. La remodelación del área

${ }^{38}$ B.N.C, Diario de la Costa. Cartagena., 21 de abril de 1956.

${ }^{39}$ A propósito de los imaginarios del miedo véase a Niño, Soledad, Territorios del miedo: imaginarios de los ciudadanos, Bogotá, TM Editores- Observatorio de Cultura Urbana, 1998. Martel, Roxana y Baires, Sonia Imaginarios del miedo y geografías de la inseguridad: construcción social y simbólica del espacio público, en: Hiernaux, (Coords.) Barcelona, Anthropos. UAM-I, 2006

${ }^{40}$ Orlando De Ávila, Op.cit, p. 128 
${ }^{41}$ Corporación Nacional de Turismo. Plan acción para el proyecto de desarrollo de turismo de la Costa Atlántic y San Andrés. Corporación Nacional de Turismo de Colombia, 1972.

${ }^{42}$ A.H.C." Debate público sobre Chambacú pide Don Antonio Dager". En: El Diario de la Costa. Septiembre 4 de 1978 , p. 2 . Las cursivas son nuestras. de la plaza de mercado localizada a l lado de la bahía y adyacente a la ciudad amurallada, se ha iniciado con la construcción de una nueva plaza de mercado en otro sitio, y se está planeando el desarrollo de esta área ${ }^{41}$.

El Mercado Público de Getsemaní, contradecía abiertamente al plan de la ciudad turística que se quería, por eso su traslado en 1978 no tendría reversa. Muchos fueron los imaginarios que trataron de legitimar tal acción, a fin de crear en este espacio, la sensación de caos, desorden y una brutal amenaza a la salud pública de los cartageneros. Los imaginarios que se constituyen desde la prensa, discursos oficiales y demás medios, afirmaban que el Mercado tendría que estar fuera del centro histórico.

Queda en evidencia que esa ciudad que afanosamente se constituía en turística, buscó cubrir a pinceladas aquellas cosas que deslucían la nueva meca del turismo nacional. Por ese gran motivo, se justificó el desaforado traslado que hasta ahora empieza a ser evaluada por los estudios sociales de la ciudad. No obstante, las posiciones del traslado no fueron homogéneas, muchos hicieron escuchar sus voces y dejaron entrever el malestar que significó haber borrado el Mercado de Getsemaní. Para septiembre de 1978, (ya sin el Mercado de Getsemaní) un distinguido habitante de la cuidad -Don Antonio Dáger - había sido uno de los más notables dirigentes pro-turísticos de la cuidad, sentó su postura crítica acerca de los planes de desarrollo urbano de predios como Chambacú y el antiguo mercado e invitó a través de la prensa a un debate público acerca de este fenómeno:

(...) sobre lo que se tiene proyectado en el sector del viejo mercado es otro de los tantos actos vandálicos que se siguen cometiendo contra nuestro patrimonio colonial. (...). El traslado del viejo mercado para Bazurto se hizo en una forma tan precipitada provocando con ello una metástasis, con graves perjuicios para los usuarios (...). Aquí se le ha dado luz verde para el proyecto elaborado para este sector que es lo más absurdo que se puede concebir ${ }^{42}$.

Las palabras casi premonitorias de Dáger, manifestaba dicho sea de paso, la actitud deliberada de la administración local, en aras de expulsar del centro turístico de la ciudad cualquier signo de pobreza, esta que en muchas ocasiones era asociada a la criminalidad, el caos y la insalubridad. Rasgos negativos y a la vez discordantes al discurso patrimonial, de la entonces 
pensada hidalga Cartagena. Estos casos como el de Chambacú y el Mercado Público de Getsemaní, se reproducen a menudo en la ciudad, pues en la actualidad se siguen adelantando nuevos proyecto de renovación urbana, los cuales están orientados a despojar del centro histórico - turístico de Cartagena, aquellos sectores incoherentes a la imagen; como bien lo señalaba Bauman ${ }^{43}$, "los extraños, parado y el basurero son antagónicos al turismo", puesto que estos solo producen molestias y echan abajo el telón de las apariencias.

\section{CONCLUSIONES}

El sector turístico de Cartagena es un proyecto de ciudad que se pensó y se construye durante buena parte del siglo XX y aún en nuestros días. La aparición de las primeras reglamentaciones orientadas hacia el cuidado de los monumentos arquitectónicos como las murallas y los demás fuertes, sirvieron de plataforma para que la ciudad comenzara a forjar un discurso turístico. La llegada cada vez más frecuentes de turistas en los años 30 y 40, ayudó a que la empresa turística fuera objeto de mayores atenciones y reglamentaciones por parte del Gobierno Nacional. En ese contexto, Cartagena se inserta dentro de estas políticas turísticas, tanto así, que en 1943 la ciudad es declarada bajo la Ley 86 como primera ciudad turística del país. Finalizando la década los 50, el desarrollo turístico de la ciudad se concentró en la promoción y adecuación espacial de la ciudad para el uso del turismo a través del cuidado y embellecimiento de los sitios de interés turístico, como también en tratar de conseguir por medio del gobierno nacional, los recursos necesarios para intervenir la estructura arquitectónica de aquellos inmuebles nacionales con los que se atraía a los turistas. No fue sino hasta los 60, cuando empiezan a restaurarse muchos de los monumentos, gracias al proyecto del historiador y restaurador español Juan Manuel Zapatero. A pesar de un dispendioso desarrollo de la ciudad en el sector turístico, gracias, entre otras cosas, a la masificación que esta empresa tiene a nivel mundial después de la posguerra, el problema que enfrentaban las autoridades pro-turísticas era la época de conciencia turística de la comunidad, especialmente los pobres que vivían o pasaban la mayor parte de su tiempo en los alrededores del centro histórico - turístico de la ciudad. El aspecto que estos sectores mostraban, al parecer, no era digno de exponer a los turistas y mucho menos, si la ciudad que se ofertaba defendía un discurso donde todo parecía estar en perfecto estado. 
Así pues, entrados los años 70, los dirigentes locales comenzarían a erradicar cualquier "zona negra" o sombra de pobreza cercana al centro histórico. Chambacú en 1971 y el Mercado Público de Getsemaní en 1978, son el más fiel de los testimonios de esta segregación y exclusión que sufrieron los sectores populares de la ciudad. A los primeros se fragmentó y segregó a las goteras de Cartagena, en barrios que hoy por hoy son en los que más se manifiesta la pobreza y el olvido estatal. El segundo, se trasladó a un gran edificio lejos del centro, donde a falta de políticas educativas, creció uno de los lugares de mayor congestión y caos de la ciudad. En Cartagena cada día se está borrando todo aquello que contradiga a la imagen turística de la ciudad. A partir de la declaración de la UNESCO como Patrimonio Histórico de la Humanidad en 1984, parece que esta lógica de expulsar a los pobres se intensificó aún más; y a pesar que Cartagena posee uno de los más renombrados patrimonios para el uso y disfrute de todos los habitantes del planeta, contradictoriamente, este no está al servicio de sus ciudadanos. En este sentido, todas aquellas discusiones y caracterizaciones teóricas a propósito de la ciudad y el turismo, nos lleva a concluir que Cartagena es una ciudad donde se ofrece un panorama de extremos inconciliables, y donde se han disuelto los lazos de cohesión social ya que los recursos parecen ser destinados exclusivamente en la infraestructura turística, patrón que por muchos años ha profundizado las desigualdades sociales y ha cambiado el paisaje urbano de la ciudad, acción en el que los sectores populares han tenido que pagar un alto precio.

\section{BIBLIOGRAFIA}

\section{Fuentes Primarias:}

Archivo Biblioteca Nacional. El Universal: "Para atraer el turismo internacional se propone crear distrito turístico" Miércoles 3 de Junio de 1963, p. 1 y 8.

A.B.N. El Universal. "Amplio plan de turismo está siendo elaborado por la Junta" Viernes 25 de febrero de 1955, N 2077, p. 1y 5.

A.B.N. EL Universal. "Vasto plan para incrementar el turismo local propone Martelo", Miércoles 17 de abril de 1963, N 5436, p. 1

A.B.N, El Universal "ECA estudia oficina de información turística solicitada por Promotora", Jueves 5 de diciembre de $1963, \mathrm{~N}^{\circ} 5023$, p. 1. 
A.B.N. El Universal. "Centro turístico tendrá Cartagena", Miércoles 24 de diciembre de 1968, N 8282 , p. 1-12.

Archivo Histórico de Cartagena. Diario Oficial. 23 de septiembre de 1932. Ley 11 de 1932, por la cual se crea en Cartagena la Junta de Monumentos históricos y de Turismo.

Corporación Nacional de Turismo. Plan de acción para el proyecto de desarrollo de turismo de la Costa Atlántica y San Andrés. Corporación Nacional de Turismo de Colombia, 1972.

\section{Fuentes Secundarias:}

Báez, Javier. Calvo, Haroldo (2000). Serie de estudios sobre la costa Caribe: balance del turismo regional en la década de los noventa. Casos de Barranquilla, Santa Marta y Cartagena. Cartagena: Departamento de Investigaciones, Universidad Jorge Tadeo Lozano.

Bernal, Camila. Quintero, Paola. López Héctor, (2005). "La competitividad turística de Cartagena de Indias: análisis del destino y posicionamiento en el mercado", en: estudios sobre la competitividad de Cartagena, serie $n^{\circ} 4$, Observatorio del Caribe.

Baumam, Zygmunt. (2001). La posmodernidad y sus descontentos. Madrid: Akal ediciones.

Carrillo Romero, Harold. (2011). Ocultar la pobreza para inventar el paraíso: la construcción de la imagen turística de Cartagena 1953 - 1978. Tesis de grado para optar al título de historiador. Cartagena: Universidad de Cartagena.

Carrillo Romero, Harold. El desarrollo de la actividad turística en Cartagena de Indias: Implicaciones Sociales y Urbanas. 1943-1978. Chicangana, Yobenh. (Comp.). 2009. Memorias VIII Encuentro Nacional de Estudiantes de Historia. Medellín: Universidad Nacional Sede Medellín.

Deavila Pertuz, Orlando. (2008). Políticas urbanas, pobreza y exclusión social en Cartagena: El caso de Chambacú 1956 - 1971, Tesis para optar el título de historiador. Cartagena: Universidad de Cartagena.

De León, Cebaldo, Pereiro, Xeraldo. (1995). La construcción imaginaria del lugar turístico: Kuna Yala. España: Universidad de Andalucía.

Gisper, Carlos. "Las consecuencias sociales del turismo", en: Gisper, Carlos. (dir.). (1993). Enciclopedia práctica profesional de turismo hoteles y restaurantes, Barcelona: Editorial Océano. 
Meisel Roca, Adolfo, y Aguilera, María Modesta. (2009). “ila islas que se repite? Cartagena en el censo de población de 2005", en: Documentos de trabajos sobre economía regional № 109, Cartagena: Banco de la República.

Meisel Roca, Adolfo. "Cartagena, 1900 - 1950: A remolque de la economía nacional”, en: Calvo Stevenson, Haroldo y Meisel Roca, Adolfo (comps.). (2000). Cartagena de Indias en el siglo XX Bogotá: Banco de la República.

Méndez, Eloy. 2008. "Imaginario del lugar", en: Topofilia: revista de arquitectura, urbanismo y ciencias sociales, número especial. México: Primer Coloquio Internacional: Ciudades del Turismo.

Niño, Soledad. (1998). Territorios del miedo: imaginarios de los ciudadanos, Bogotá: TM EditoresObservatorio de Cultura Urbana.

Paniagua, Raull. (1994). "El turismo en Cartagena", en: Díaz de Paniagua, Rosa (comp.). Cartagena popular: aproximación al análisis socio-cultural. Cartagena: Centro de Cultura Afrocaribe. pp. 113125.

Samudio, Trallero Alberto. (1994). El crecimiento urbano de Cartagena en el Siglo XX: Manga y Bocagrande, Cartagena: Universidad Jorge Tadeo Lozano.

Sierra Anaya, Germán. Marrugo. Dennise. Quejada, Pérez Raúl. (2004). La actividad del turismo en Cartagena de Indias. Cartagena: Universidad de Cartagena, Facultad de Ciencias Económicas.

Sierra Anaya, Germán. (1998). Viajeros y visitantes. Una historia del turismo de Cartagena de Indias. 1501-1959. Cartagena: Heliógrafo moderno.

Vidal, Claudia. (1998). Los inicios de la industria turística en Cartagena, 1900-1950, Tesis de grado para optar al título de historiadora. Cartagena: Universidad de Cartagena. 


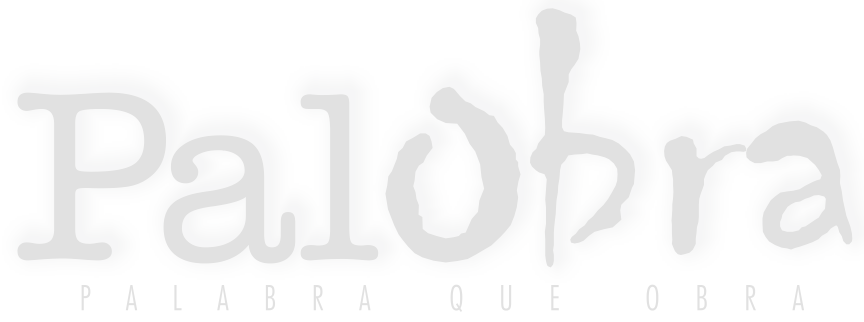

